\title{
Integrated metagenomics and metabolomics analysis of third- trimester pregnant women with premature membrane rupture: a pilot study
}

\author{
Lou Liu ${ }^{1 \#, ~ Y u ~ C h e n ~}{ }^{2 \#}$, Jia-Le Chen ${ }^{3 \#}$, Han-Jie Xu ${ }^{4}$, Hui-Ying Zhan ${ }^{1}$, Zhong Chen ${ }^{1}$, Dao-Zhen Chen ${ }^{1}$, \\ Zheng-Feng $\mathrm{Xu}^{5}$, De-Xiang $\mathrm{Xu}^{3}$
}

${ }^{1}$ The Affiliated Wuxi Maternity and Child Health Care Hospital of Nanjing Medical University, Wuxi, China; ${ }^{2}$ Research Institute for Reproductive Health and Genetic Diseases, The Affiliated Wuxi Maternity and Child Health Care Hospital of Nanjing Medical University, Wuxi, China; ${ }^{3}$ The School of Public Health, Anhui Medical University, Hefei, China; ${ }^{4}$ The School of Wuxi Clinical Medicine Nanjing Medical University, Nanjing, China; ${ }^{5}$ Center of Medical Genetics, Obstetrics and Gynecology Hospital Affiliated to Nanjing Medical University, Nanjing, China Contributions: (I) Conception and design: L Liu, DZ Chen, ZF Xu, DX Xu; (II) Administrative support: DZ Chen, ZF Xu; (III) Provision of study materials or patients: Z Chen, HY Zhan; (IV) Collection and assembly of data: L Liu, Y Chen; (V) Data analysis and interpretation: L Liu, JL Chen; (VI) Manuscript writing: All authors; (VII) Final approval of manuscript: All authors.

\#These authors contributed equally to this work.

Correspondence to: Dao-Zhen Chen. 48 Huaishu Alley, Zhong Shan Road, Wuxi 214002, China. Email: chendaozhen@163.com; Zheng-Feng Xu. No. 123, Tianfei Lane, Mochou Road, Qinhuai District, Nanjing 210004, China. Email: njxzf@126.com; De-Xiang Xu. 81 Meishan Road, Hefei 230032, China. Email: xudex@126.com.

Background: Premature rupture of membranes (PROM) is a major pregnancy complication in China and usually leads to adverse pregnancy outcomes. The major aim of this study was to search for microorganisms and their related metabolites that have direct relationship with PROM.

Methods: For vaginal discharge samples, metagenomics sequencing was applied to identify microorganisms that were enriched in PROM subjects, and untargeted metabolomics was applied to characterize the metabolites changes in PROM subjects compared to healthy controls (HC). Correlation analysis was then used to explore the relationship between these microorganisms and metabolites changes.

Results: Two upstream metabolites of glycolysis, N-acetyl-D-galactosamine (GalNAc) and sucrose, were found downregulated in the PROM group ( $\mathrm{P}=0.04$ and $\mathrm{P}=0.041$, respectively). Higher percentages of conditional pathogens, such as of Streptococcus (8.4\% vs. 6.1\% in HC group, $\mathrm{P}=0.15)$ and Chlamydia ( $4.3 \%$ vs. $2.3 \%$ in HC group, $\mathrm{P}=0.07$ ) were found in PROM group. Other common conditional pathogens including Prevotella, Staphylococcus, Mycobacterium and Enterobacter, were also higher in PROM group, although their absolute percentages were low and the differences did not reach statistical significance due to relative small sample size. Correlation analysis further demonstrated a positive correlation of downregulation of glycolysis metabolites with higher percentage of conditional pathogens.

Conclusions: Integrated metagenomics and metabolomics analysis can be used to track the subtle changes in the vaginal microenvironment. Downregulation of glycolysis substrates (GalNAc and sucrose) and increase of related pathogenic microorganisms (Streptococcus and Chlamydia) could serve as early warning biomarkers of PROM.

Keywords: Premature rupture of membranes (PROM); metabolomics; metagenomics; vaginal dysbiosis; glycolysis

Submitted Sep 10, 2021. Accepted for publication Nov 19, 2021.

doi: 10.21037/atm-21-5539

View this article at: https://dx.doi.org/10.21037/atm-21-5539 


\section{Introduction}

Premature rupture of membranes (PROM) refers to spontaneous rupture of membranes occurring before the onset of labor. If fetal membrane rupture happens before 37 gestational weeks, it is defined as pre-PROM (pPROM), which is a common cause of preterm labor and usually leads to massive medical expenses (1). Interestingly, the incidence of PROM in China (15.3\%) (2) has been reported to be higher than that of preterm labor (7\%) (3), which is opposite to the trend seen in Western countries, and is likely due to the differing definitions of preterm labor. In China, preterm delivery is defined as the birth of an infant after 28 but before 37 weeks of gestation, while in Western countries or in higher resource settings, it usually refers to delivery between 23-24 and 37 weeks.

Antibiotic-resistant infection is extremely common in China. Resistance to antibiotics can lead to the occurrence of reproductive tract infections caused by conditional pathogens, such as chorioamnionitis, fetal infection, and neonatal sepsis, during the time between membrane rupture and neonatal birth, when the balanced state between pathogen and host is broken. PROM is often accompanied by ascending genital infection (4), which can result in severe complications of pregnancy. A growing number of studies show that disruption of the normal vaginal microecology caused by compositional changes in the vaginal microbiome increases the morbidity of preterm birth and PROM (5). Vaginal microecological disorders can disturb the ecobalance of vaginal flora, and the conditional pathogens responsible usually proliferate rapidly, resulting in ascending genital infection.

According to previous studies, bacterial vaginitis (BV) is the major cause of genital infection, and its common pathogens include Candida albicans, trichomonads, and Gardnerella. These microorganisms lead to ascending genital infection and inflammation. Inflammatory cytokines released during inflammation response usually result in reduced tension of fetal membrane, and therefore increase the risk of developing PROM. During pregnancy, BV is usually diagnosed and treated properly during routine examinations; however, some pregnant women develop $\mathrm{BV}$ recurrence (6). In such cases, traditional laboratory tests, including leukorrhea screening and vaginal secretion culture, are performed, and the Nugent score is regular checked during the pregnancy. However, these methods can only detect less than $1 \%$ of pathogens in the vagina (7). Furthermore, laboratory results sometimes cannot determine whether the pathogens detected are actively proliferating, and some anaerobic bacteria and facultative anaerobes cannot be successfully cultured on the traditional Petri dish, meaning vaginal secretion culture may not be able to cover all species of pathogens. Moreover, approximately $30-50 \%$ of patients with BV do not have clinical symptoms such as increased leukorrhea or abnormal odor (8). Therefore, a large proportion of pregnant women who suffer from asymptomatic vaginal infection may not be effectively diagnosed by culture at a very early stage.

With the development of new detection technologies, an increasing number of microorganism species and metabolites have been shown to be related to PROM. In recent years, culture-independent omics technology has been applied to analyze and cluster pathogens in human microbiota (9). Therefore, omics technology may be also used to identify vaginal microorganism composition and clarify their contribution to PROM development. Based on the findings from omics-based testing, targeted preventive treatment can be applied to prevent adverse pregnancy outcomes after PROM (10). More specifically, metagenomics may be used to figure out the detailed microorganism composition in the lower genital tract and compare differences in microorganism composition under normal and vaginal infection conditions. At the same time, metabolomics may be used to identify the metabolites associated with microorganism changes and to study how these pathogens destroy the vaginal microenvironmental balance. For example, Paramel Jayaprakash et al. (11) reported a prospective cohort study of vaginal microbiome in pPROM subjects using metagenomic approach.

Brown et al. (12) studied vaginal microorganism composition at four pregnancy stages $\left(12-17^{+6}, 18-23^{+6}\right.$, $24-29^{+6}$, and $30-36^{+6}$ gestational weeks), and found that vaginal dysbiosis at $24-29^{+6}$ and $30-36^{+6}$ weeks significantly increased the risk of pPROM; the authors subsequently termed these two stages as "immune clock". Earlier studies have found that about $30 \%$ of healthy pregnant women's vaginal flora is in a state of lack of Lactobacillus, however there are no signs of disease (13). We believe the interaction/cross-talk between the microorganisms and the host plays an important role in the development of PROM (14). Therefore in addition to Jayaprakash's study, we designed a pilot study using an integrated metagenomics and metabolomics approach in an effort to better understand the relationship between microorganism composition and metabolites in the vagina, and illuminate the potential mechanism of how lower genital infection may induce PROM in the third trimester of pregnancy (from 31 
to 36 gestational weeks). We present the following article in accordance with the MDAR reporting checklist (available at https://dx.doi.org/10.21037/atm-21-5539).

\section{Methods}

\section{Subjects}

From January 1, 2019 to February 28, 2020, 163 pregnant women were recruited from the Obstetric Department of the Affiliated Wuxi Maternity and Child Health Care Hospital of Nanjing Medical University. This study was approved by the Ethics Committee of the Affiliated Wuxi Maternity and Child Health Care Hospital of Nanjing Medical University (No. 2019-02-0402-01) and was conducted in adherence with the Declaration of Helsinki (as revised in 2013). The study was retrospectively registered in the Chinese Clinical Trial Registry (ChiCTR2000034721, registration date: 2020-7-16). Written informed consent was obtained from each participant.

All the study participants met the following criteria: (I) women with singleton pregnancy, with cephalic presentation; (II) received regular prenatal examination; and (III) delivered vaginally. Pregnant women with any of the following conditions were excluded from the study: (I) complications which may disturb the study, such as preeclampsia, gestational diabetes, malnutrition, multiple gestation, and breech presentation; (II) a short cervical length; and (III) a preterm labor history, Pregnant women with oral or vaginal medicine administration, sexual activity or vaginal douching within 48 hours before receiving the swab were also excluded.

\section{Prenatal examination and delivery}

All study participants underwent regular prenatal examination according to the World Health Organization's antenatal care guidelines (i.e., initial examination within the first 12 gestational weeks, with subsequent follow-ups at $20,26,30,34,36,38$, and 40 gestational weeks). Vaginal swabs were collected at the posterior fornix between 31 and 36 weeks of gestation, and the swabs were stored at $-80{ }^{\circ} \mathrm{C}$ immediately after sampling. Two samples were collected per participant at the same time: one sample for untargeted metabolomics detection and the other for metagenomic sequencing. All study participants delivered their babies in the Affiliated Wuxi Maternity and Child Health Care Hospital of Nanjing Medical University.

\section{Selection of PROM cases and healthy controls $(H C)$}

PROM was diagnosed according to the "ACOG Practice Bulletin No. 188: Prelabor Rupture of Membranes" guidelines (15). Pregnant women who met the criteria for PROM were assigned to the PROM group, while pregnant women with healthy vaginal delivery were assigned to the HC group. After all the participants had delivered their babies, samples from the PROM and HC groups were selected for further analysis using the computer-generated random number sampling method. Considering the research budget and the representativeness of the samples, six samples from the PROM group and five samples from the HC group were ultimately selected for metagenomics and metabolomics research.

\section{Untargeted metabolomics detection}

Untargeted liquid chromatography-tandem mass spectrometry (LC-MS/MS, BGI Genomics, China) was performed to detect metabolites in swab samples from the selected pregnant women. Briefly, the vaginal swabs were weighed and eluted in methanol-water $(1: 1)$ to a final concentration of $50 \mathrm{mg}$ vaginal fluid/ml. The samples were then microfiltered and passed through an Agilent 1290 Infinity HPLC system coupled with a Q-Exactive Orbitrap mass spectrometer (Thermo Fisher Scientific). All samples were analyzed by full MS scanning between the ranges of $50-750 \mathrm{~m} / \mathrm{z}$ in both the positive ion and negative ion modes at 140,000 sharpness of separation. A separate LCMS method was adopted for relative quantification with increased sensitivity.

The raw MS data were transferred to and further analyzed with metaX. Metabolites were identified and annotated using Compound Discoverer 3.0 (Thermo Fisher Scientific, USA) through BGI (Beijing Genomics institution) group, mzCloud, and Chemspider libraries. Differentially expressed metabolites between the PROM and HC groups were determined based on the following criteria: (I) partial least squares discriminant analysis model (VIP $\geq 1$ ); (II) fold change $\geq 1.2$ (or $\leq 0.83$ ); and (III) $\mathrm{P}$ value $<0.05$. Eighty human metabolic pathways in the Kyoto Encyclopedia of Genes and Genomes (KEGG) database served as reference metabolic pathways. To better elucidate the functional impact of vaginal metabolites in PROM, a differentially expressed metabolite heatmap and metabolic pathway enrichment analysis were done using the MetaboAnalyst 3.0 computational platform. In pathway 
enrichment analysis, a single $\mathrm{P}$ value was computed for each metabolic pathway (a group of function-associated metabolites).

\section{Metagenomics sequencing}

Vaginal swab DNA samples were extracted using the FastDNA Spin extraction kit (MP Biomedicals, Santa Ana, CA, USA). The DNA samples were then broken down by sonication, and the Agencourt AMPure XP-Medium kit was used to enrich short DNA fragments (300-400 bp) for PCR amplification and library preparation. All of the samples were sequenced by Huada Gene Laboratory using rolling circle replication and a DNA nanoball sequencing method.

Raw sequencing data were filtered to remove the sequences from host human genome through Sopa2 alignment. Subsequently, the sequences were assembled de novo using the IDBAUD assembly software. Open reading frames (ORFs) were predicted using Metagenemark (version 2.10, default parameters, http://exon.gatech.edu/ GeneMark/metagenome/Prediction/). The high-quality reads from different samples were aligned to the established gene set using bowtie 2 and annotated based on public databases.

\section{Correlation analysis}

The correlation analysis aimed to combine differential metabolites and differential microorganism species into a comprehensive analysis covering several aspects, including quantitative correlation, functional correlation, and phenotypic correlation. The typical methods used included Spearman's rank correlation, canonical correlation analysis (CCA), the Mantel test, pathway enrichment, and microorganism KEGG module enrichment. The flow chart for the metagenomics and metabolomics correlation analysis is shown in Figure S1.

\section{Statistical analysis}

Continuous variables were expressed as medians with ranges (min-max). Differences between the groups were analyzed by $t$-test. Categorical variables were presented as numbers and analyzed by the chi-square test. All analyses were performed in $\mathrm{R}$ for Windows (version 20.0; IBM Inc, Chicago, IL, USA), with a two-tailed $\mathrm{P}$ value of $<0.05$ considered statistically significant.

\section{Results}

\section{Demographical and clinical data}

A total of 163 pregnant women were initially recruited in the study, of whom 36 developed PROM. Due to the outbreak of COVID-19, 40 participants were lost to followup and were thus excluded from the calculation of PROM incidence (29.3\%). In an earlier study, we chose 20 pregnant women from the present study cohort (10 with PROM and $10 \mathrm{HCs}$ ) for metabolomics analysis (16). From these 20 women, 6 PROM cases and 5 HCs were further selected at random for the current metagenomics and metabolomics coupling study. Their clinical characteristics, including age, nulliparity, white blood cell count at admission, positive genital culture at admission, and gestational age at sampling, were well matched to avoid artificial disturbance of the results. None of the pregnant women received steroid administration during pregnancy. There were no statistical differences in tocolysis treatment, gestational age at delivery, latency from sampling to delivery, or baby weight or sex at birth between the PROM and HC groups. Overall, the metagenomics and metabolomics data of the two groups in this study were comparable (Table 1).

\section{GalNAc and sucrose are downregulated in patients with PROM}

To identify vaginal metabolites which are related and contribute to the development of PROM, we first applied a metabolomics approach to analyze samples from the PROM and $\mathrm{HC}$ groups. In the PROM samples, 82 metabolites were statistically significantly upregulated or downregulated (Figure S2). Through investigating GalNAc and sucrose on the KEGG: Kyoto Encyclopedia of Genes and Genomes, and searching Glycolysis/Gluconeogniesis from KEGG PATHWAY: Glycolysis/Gluconeogenesis-Reference pathway, interestingly, we found GalNAc and sucrose, which are two important sources for glycolysis (Figure S3), were both downregulated in the PROM group $(\mathrm{P}=0.04$ and $\mathrm{P}=0.041$, respectively, Figure 1). This result is consistent with the conclusion of our earlier study (16). Lactobacillus microbes which normally exist in the vagina are well known to rely on glycolysis to produce lactic acid and maintain the correct vaginal $\mathrm{pH}$ environment. Our finding that the levels of GalNAc and sucrose were significantly lower in the PROM group than in the HC group strongly suggested that the glycolysis pathway was not sufficiently fueled in pregnant 
Table 1 Clinical characteristics of the study participants ${ }^{2}$

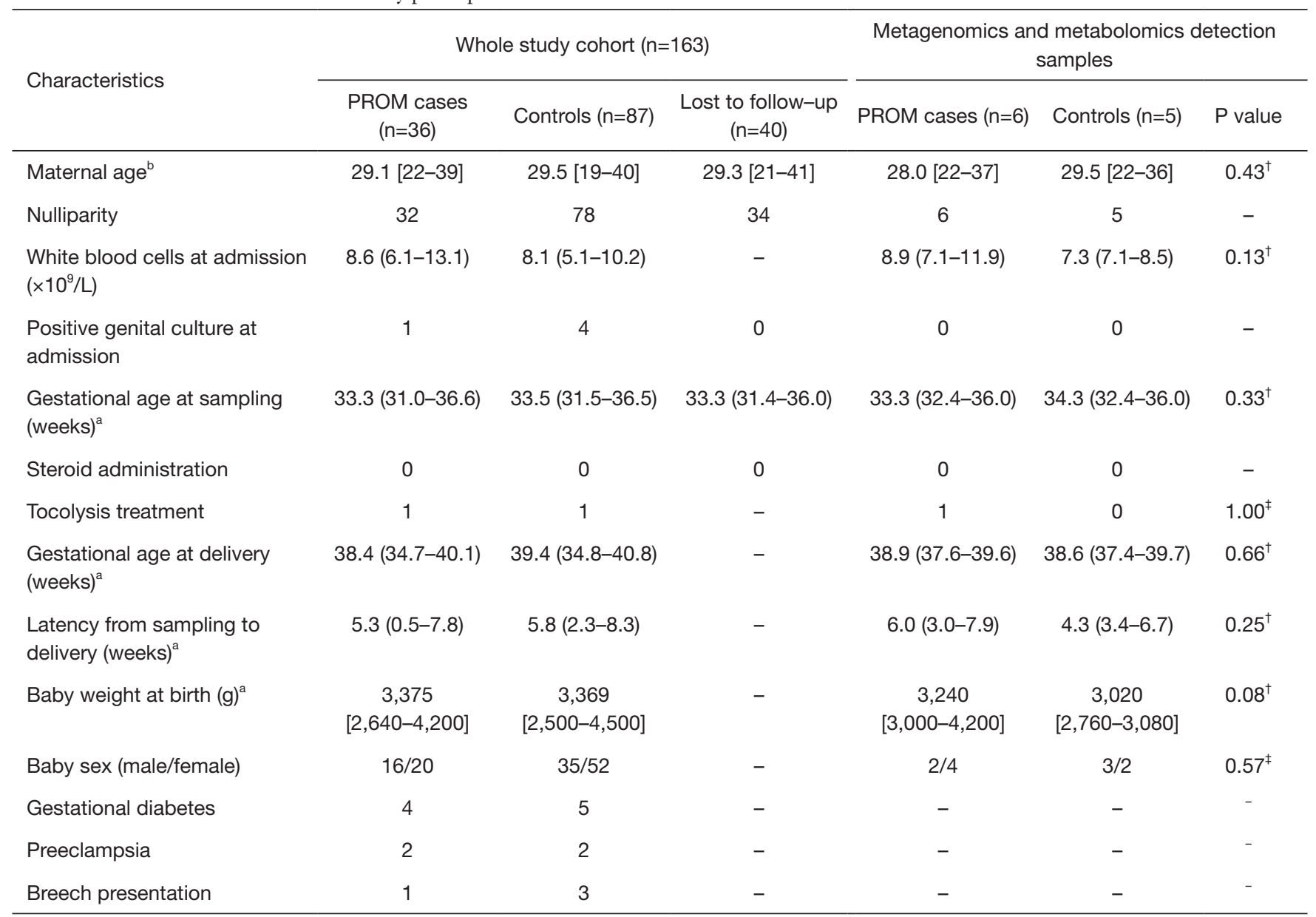

a , adapted from (16). (https://link.springer.com/article/10.1007\%2Fs43032-020-00338-9). Such adaption is permitted under a Creative Commons Attribution 4.0 International License (http://creativecommons.org/licenses/by/4.0/). ${ }^{\mathrm{b}}$, values are presented as median (minmax); ${ }^{\dagger}$, independent-sample Mann-Whitney $U$ test; ${ }^{\ddagger}$, Fisher's exact test. PROM, premature rupture of membranes.
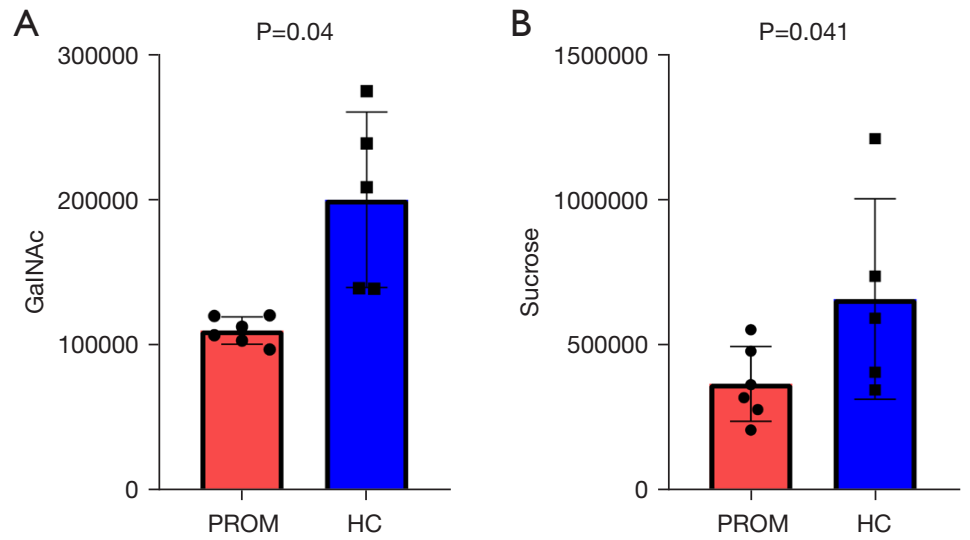

Figure 1 The upstream metabolites of glycolysis are downregulated in patients with PROM. Metabolomics analysis results showing downregulation of both GalNAc (A) and sucrose (B) in the PROM group ( $\mathrm{P}=0.04$ and $\mathrm{P}=0.041$, respectively). $\mathrm{PROM}$, premature rupture of membrane; GalNAc, N-acetyl-D-galactosamine. 
A

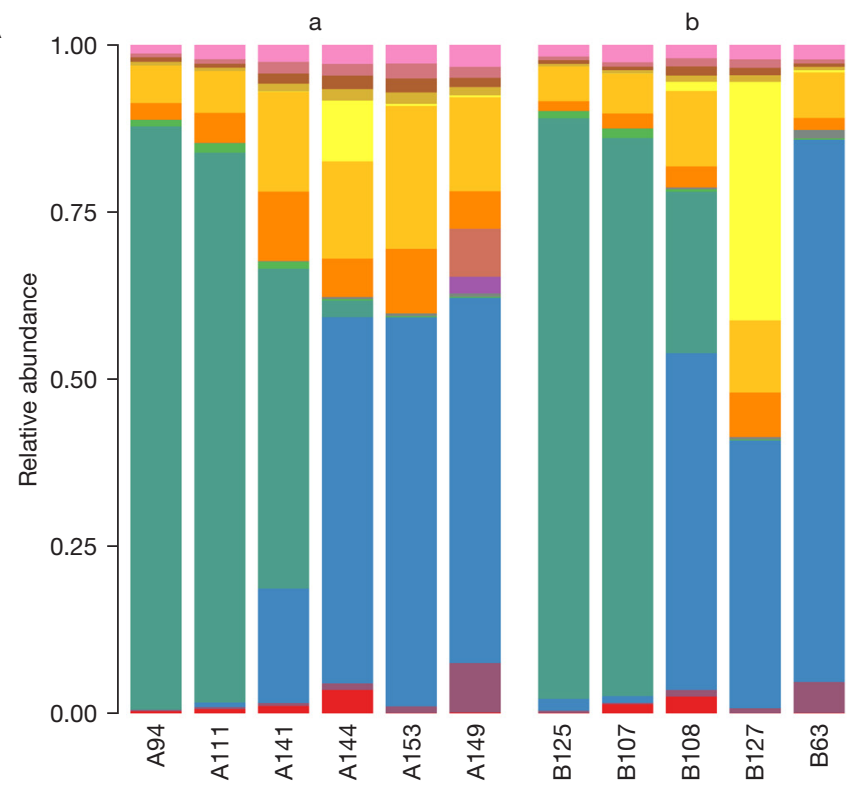

Species

Lactobacillus jensenii Lactobacillus gasseri Lactobacillus iners Lactobacillus crispatus Lactobacillus helveticus Lactobacillus sp.7147FAA Prevotella bivia Prevotella disiens Chlamydia trachomatis Streptococcus pneumoniae Gardnerella vaginalis Enterobacter cloacae complex Streptococcus aureus Mycobacterium tuberculosis complex Others

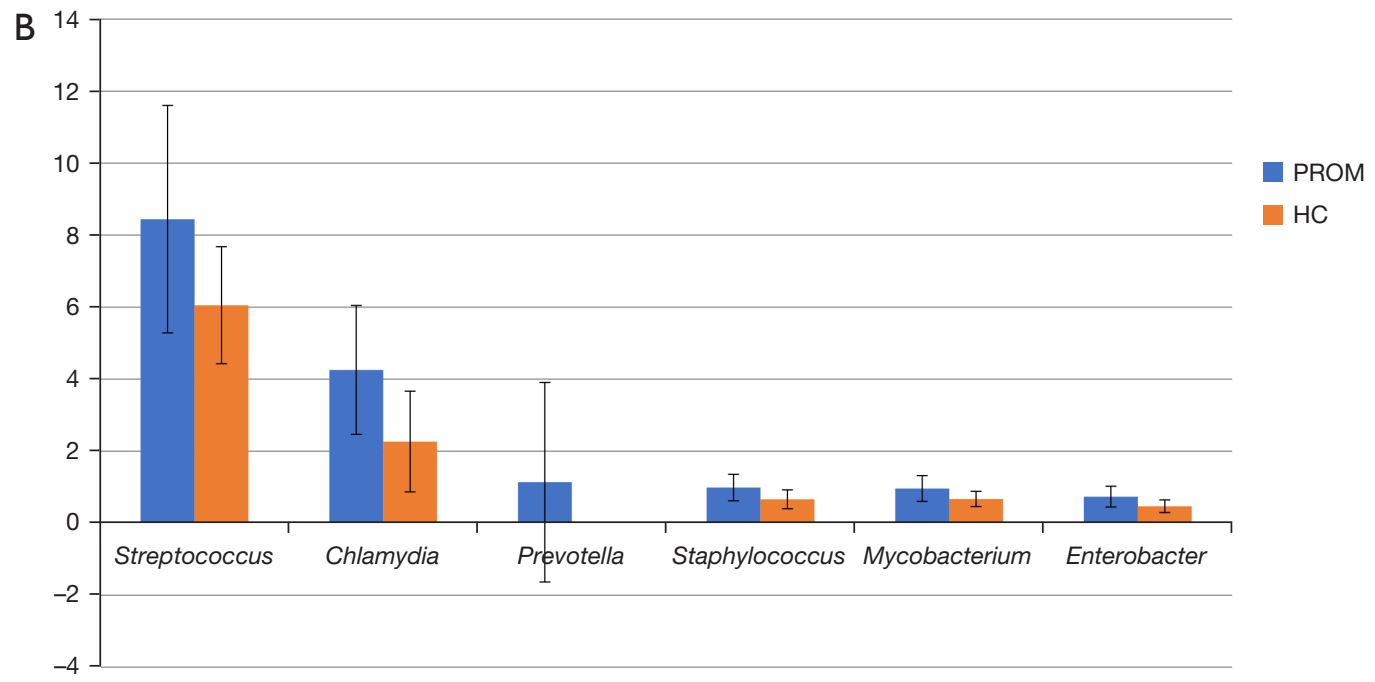

Figure 2 The percentages of certain conditional pathogens are higher in patients with PROM. (A) Microorganism composition of six PROM samples and five HC samples. (B) The percentages of six major conditional pathogens were higher in the PROM group than the HC group, although the difference did not reach statistical significance. Streptococcus, $\mathrm{P}=0.15$; Chlamydia, $\mathrm{P}=0.069$; Prevotella, $\mathrm{P}=0.36$; Staphylococcus, $\mathrm{P}=0.12$; Mycobacterium, $\mathrm{P}=0.13$; Enterobacter, $\mathrm{P}=0.096$. PROM, premature rupture of membrane; HC, healthy control.

women with PROM. In this scenario, Lactobacillus microbes might not be able to produce sufficient lactic acid to suppress the proliferation of pathogenic microorganisms, eventually leading to the development of BV, and eventually PROM.

\section{The percentages of certain conditional pathogens are bigher in patients with PROM}

Due to the limited sample number and huge variation among individual samples in this study, we failed to identify a clear relationship between microorganism composition and PROM development (Figure 2A). However, the percentages of certain conditional pathogens were found to be higher in the PROM group than in the HC group. For instance, the percentage of Streptococcus in the PROM and $\mathrm{HC}$ groups was $8.4 \%$ and $6.1 \%$, respectively $(\mathrm{P}=0.15)$, and the percentage of Chlamydia was $4.3 \%$ and $2.3 \%$, respectively $(\mathrm{P}=0.069)$. Furthermore, other common 
conditional pathogens (including Prevotella, Staphylococcus, Mycobacterium, and Enterobacter) were also detected at slightly higher levels in the PROM group than in the HC group, although the percentages of these pathogens were low (approximately 1\%) (Figure 2B). However, the percentage of Lactobacillus, including the four most common vaginal species (Lactobacillus crispatus, Lactobacillus iners, Lactobacillus gasseri, and Lactobacillus jensenii), was lower in the PROM group than in the HC group $(50.7 \%$ vs. $60.4 \%, \mathrm{P}=0.39$ ). Overall, we observed higher percentages of common conditional pathogens in the PROM group than in the HC group, although the difference failed to reach statistical significance.

\section{Correlation analysis identified differentially expressed enzymes and KEGG orthology modules between the two groups}

To determine whether the small changes in microorganism composition between the PROM and HC groups has a significant effect on biological function outcomes, we carried out correlation analysis of metagenomics and metabolomics data. There was a statistically significant difference in KEGG orthology $(\mathrm{KO})$ module enrichment between the PROM and HC groups. The Shannon index is usually used to measure the Alpha diversity of individual samples. There was no significant difference in the Shannon index between the PROM and HC group samples at the species level (data not shown), which was consistent with the absence of a significant difference in microorganism composition. However, at the KO level, the Shannon index showed a significant difference between the two groups (3.092 in the PROM group vs. 3.792 in the HC group, $\mathrm{P}=0.0098$, Figure $3 A$ ), which indicated that some biological pathways in the PROM group were disrupted. A total of 15 enzymes with statistically significant differential expression were identified between the PROM group and the HC group. These enzymes mainly play important roles in threonyltRNA synthesis and glycosis, starch, sucrose, and galactose metabolism (Figure 3B). Interestingly, correlation analysis of the biological functions of the microorganisms with compositional differences showed that all four common vaginal Lactobacillus species (Lactobacillus jensenii, Lactobacillus crispatus, Lactobacillus iners, and Lactobacillus gasseri) were enriched and had biological functions in the HC group; in contrast, the PROM group had no Lactobacillus biological function enrichment (Figure 3C).

\section{Vaginal metabolite diversity is correlated with microorganism diversity}

Finally, we conducted correlation analysis of the 82 differentially expressed metabolites with major microorganism genera identified in samples from the PROM and HC groups. In contrast to Lactobacillus, six conditional pathogens (Prevotella, Streptococcus, Chlamydia, Mycobacterium, Staphylococcus, and Enterobacter) were clustered well. A total of 27 differentially expressed metabolites were positively or negatively correlated with the 6 conditional pathogens or Lactobacillus. The glycolysis source metabolite GalNAc showed a positive correlation with Lactobacillus but a negative correlation with the six conditional pathogens (Lactobacillus, $\mathrm{P}=0.214$; Streptococcus, $\mathrm{P}=0.121 ;$ Chlamydia, $\mathrm{P}=0.021 ;$ Prevotella, $\mathrm{P}=0.129$; Staphylococcus, $\mathrm{P}=0.009$; Mycobacterium, $\mathrm{P}=0.04$; Enterobacter, $\mathrm{P}=0.028$; Figure 4). Sucrose showed a similar correlation pattern, although it did not reach statistical significance. Together, these results strongly support our hypothesis that these conditional pathogens compete with vaginal Lactobacilli for glycolysis sources, leaving the Lactobacilli unable to produce enough lactic acid to maintain a healthy vaginal $\mathrm{pH}$ environment. Through this mechanism, the pathogens disrupt the ecobalance in the vagina, which eventually results in more severe infection and PROM.

\section{Discussion}

A total of 163 pregnant women were recruited into this prospective cohort study, of whom 36 women developed PROM. The incidence of PROM (29.3\%) in the present cohort was higher than that reported in another study (15.3\%) (2), which is possibly due to our hospital being the foremost regional hospital specializing in obstetrics and gynecology and admitting many pregnant women with complications. From the enrolled cases, we randomly selected six cases with PROM and five HCs for further metagenomics and metabolomics analysis. We ensured there were no statistical differences between the PROM and $\mathrm{HC}$ groups in clinical characteristics including maternal age, nulliparity, white blood cell count at admission, positive genital culture at admission, steroid administration, and tocolysis treatment, which have the potential to impact pregnancy outcome or increase the risk of PROM.

In our previous study, we examined vaginal metabolite differences between patients with PROM and HCs, and found that two source metabolites in the glycolysis pathway, 
A

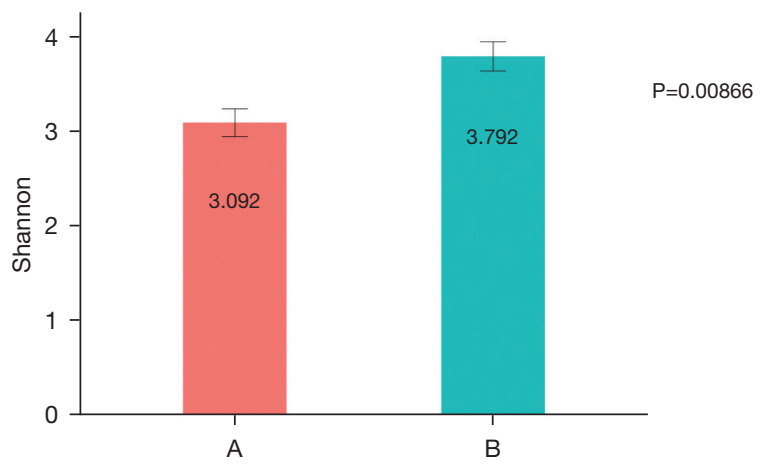

B

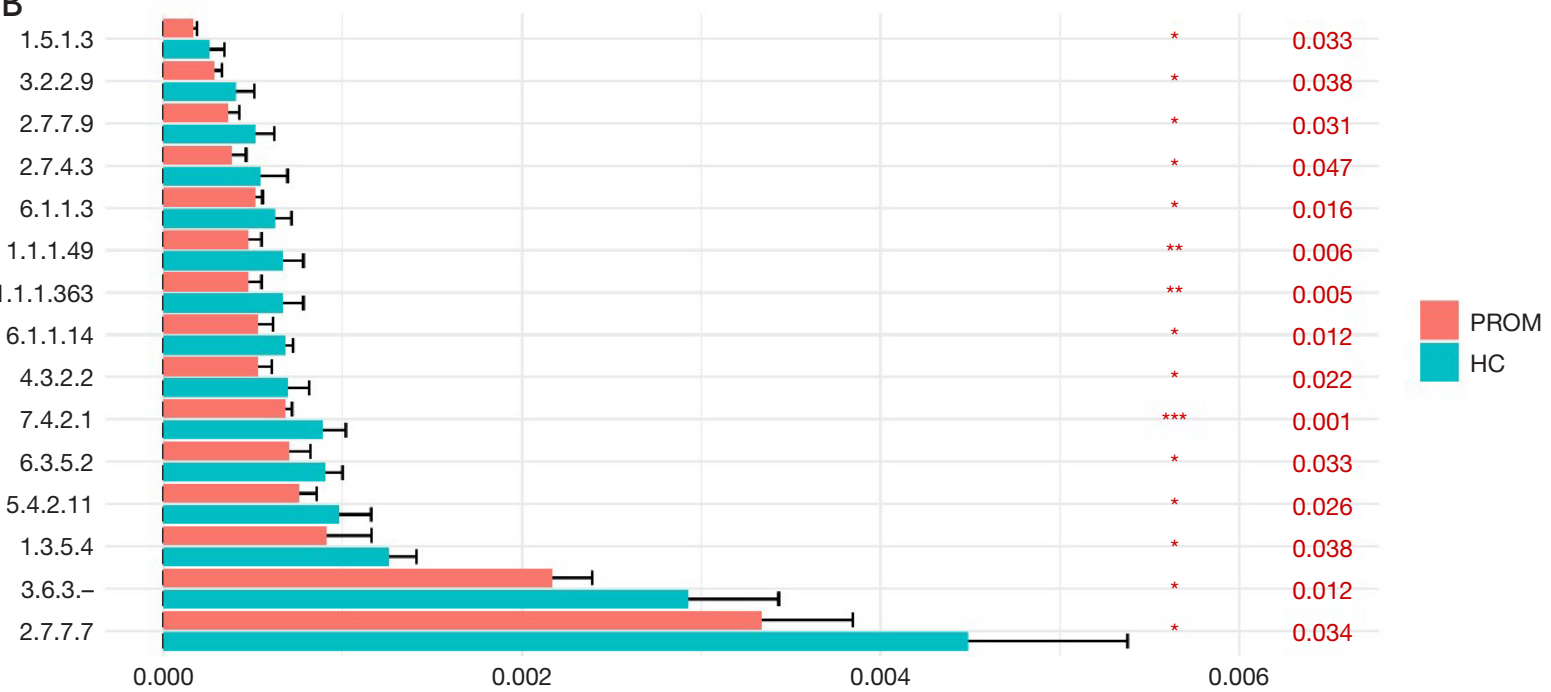

C

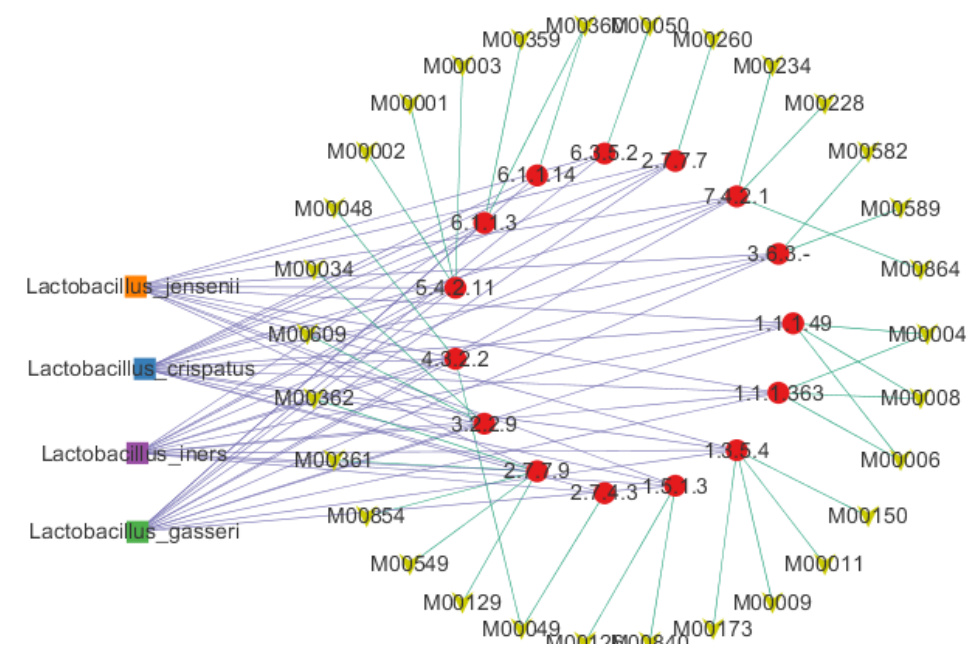

Figure 3 Correlation analysis of differentially expressed enzymes and KEGG orthology modules. (A) The Shannon index at the KEGG orthology $(\mathrm{KO})$ level showed a significant difference $(\mathrm{P}=0.0098)$. (B) A total of 15 differentially expressed enzymes were identified between the PROM and HC groups. Phosphoglycerate mutase (5.4.2.11) is an important enzyme in glycolysis; UTP-glucose-1-phosphate uridylyltransferase (2.7.7.9) participates in galactose, sucrose, and starch metabolism. (C) Correlation analysis of differentially expressed enzymes, KO modules, and microorganism species. Red dots represent differentially expressed enzymes, and yellow arrowheads represent differentially expressed KO modules. Four Lactobacillus species were enriched in the correlation analysis. KEGG, Kyoto Encyclopedia of Genes and Genomes; KO, KEGG orthology; PROM, premature rupture of membrane; HC, healthy control. 


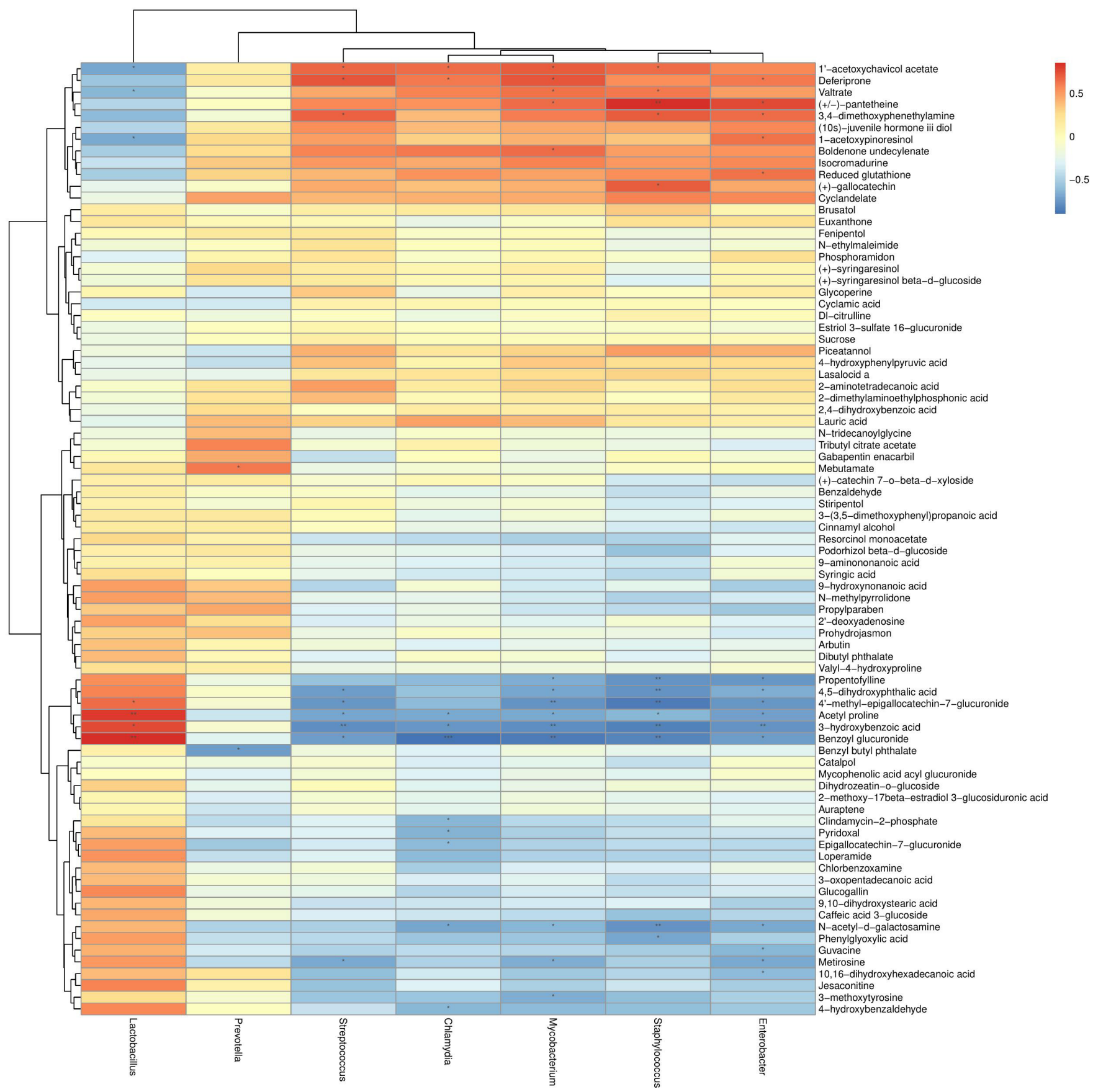

Figure 4 Vaginal metabolite diversity is correlated with microorganism diversity. Heatmap showing the correlation between microorganisms and 82 differentially expressed metabolites $\left({ }^{*}, \mathrm{P}<0.05 ;{ }^{* *}, \mathrm{P}<0.01 ; * * *, \mathrm{P}<0.001\right)$. GalNAc is positively correlated with Lactobacillus and negatively correlated with six conditional pathogens. GalNAc, N-acetyl-D-galactosamine.

GalNAc and sucrose, were downregulated in the PROM group (16); data from the current study is consistent with this finding (Figure 1). Bacteria can utilize multiple forms of sugars for their ATP production through glycolysis.
GalNAc and sucrose are the two important upstream metabolites and fuel source of glycolysis pathway. We observed that both of these two metabolites were low in our PROM group. This observation well reflected the 
scenario that proliferating pathogenic bacteria consumed a large amount of sugar source in the vaginal dysbiosis microenvironment and resulted in low supply of GalNAc and sucrose to healthy Lactobacillus species (17). However, we failed to identify statistically significant differences in individual microorganism composition between the PROM and $\mathrm{HC}$ groups, which is likely due to the study's small sample size. Nevertheless, we observed that the percentages of major conditional pathogens, such as Streptococcus, Chlamydia, Prevotella, Staphylococcus, Mycobacterium, and Enterobacter, were higher in the PROM group. This result is consistent with our observations that there is huge diversity of pathogenic microbial species in individual patients. In most cases, the mixed asymptomatic vaginal infection can further ascend to up-reproductive system and eventually lead to the development of PROM $(18,19)$.

Many previous studies have demonstrated that group B Streptococcus can lead to adverse pregnancy outcomes including genital infection and PROM (20-22). One systematic review and meta-analysis suggested that Chlamydia trachomatis could cause preterm labor and PPROM (23). Marconi et al. used enzyme-linked immunosorbent assay to analyze the levels of interleukin (IL)-1 $\beta$, IL-6, IL-8, and tumor necrosis factor (TNF)- $\alpha$ in women with intra-amniotic infection who experienced preterm labor caused by PROM, and found that most of these infection indicators were increased (24). Interestingly, Zurek et al. uncovered a mechanism by which Staphylococcus aureus could inhibit the early neutrophil-derived IL-8 response and enhance infection (25). Another study on sepsis-associated neonatal mortality in developing countries reported that $3.2 \%$ of neonates with a maternal history of PROM developed early-onset neonatal sepsis, with group B streptococcus and Staphylococcus aureus positively detected (26). It is clear that Streptococcus, Chlamydia, and Stapbylococcus are strongly related to infection and PROM in pregnancy. To our knowledge, Enterobacter is the most common microorganism found in the maternal genitourinary tract and the neonate body surface in the clinical setting. The mortality of neonatal sepsis increases in prolonged PROM, and so Enterobacter infection, which usually derives from the maternal genitourinary tract, should be prevented (27).

Through KO cluster analysis, multiple pathways and modules with significant differences between the PROM and HC groups were identified. It is worth noting that glycolysis is an extremely important pathway in the development of PROM. According to our correlation analysis of the microorganisms, enzymes, modules, and pathways with significant differences between the PROM and HC groups, various Lactobacillus species may have a protective effect against PROM development via glycolysis and lactic acid production.

Typically, four Lactobacillus species are dominant in the vagina and maintain normal vaginal microorganism composition and $\mathrm{pH}<4.5$ (28). The current classification of vaginal microecology focuses on Lactobacillus species and is divided into five community state types (CSTs). CSTs I, II, III, and V are dominated by Lactobacillus crispatus, Lactobacillus gasseri, Lactobacillus iners, and Lactobacillus jensenii, respectively, while CST IV is characterized by a low abundance of lactic acid-producing bacteria and increased diversity and abundance of anaerobic bacteria, and is usually associated with BV (13). During the development of asymptomatic vaginal infection, pathogens interact with the human vaginal mucosal epithelium immune system. If the immune response is successful and the inflammation is cleared, the infection is reversed and the fetal membranes remain intact. The local protection offered by Lactobacilli during this process is extremely important. Lactobacilli can use host $\alpha$-amylase to hydrolyze glycogen into maltodextrin, maltotriose, and maltose in vaginal fluid $(29,30)$, and analysis of the metabolite profile of vaginal mucosa showed that the concentration of maltodextrin, maltotriose, and maltose is positively correlated with the abundance of Lactobacillus crispatus and Lactobacillus jensenii. In patients with BV, the level of $\alpha$-amylase is low, and the above-mentioned carbohydrate metabolites are reduced, which is not conducive to establishing healthy local flora (31). In this study, KO correlation analysis showed that Lactobacillus jensenii, Lactobacillus crispatus, Lactobacillus iners, and Lactobacillus gasseri were all enriched in the HC group; therefore, Lactobacillus may play a protective role against the development of PROM.

In the correlation analysis between microorganism species and metabolites, many species were positively or negatively correlated with metabolites. For example, GalNAc and sucrose had positive correlations with Lactobacillus but negative correlations with six conditional pathogens. Lactobacilli play a critical role in maintaining $\mathrm{pH}<4.5$ in the vagina through the production of lactic acid to inhibit the growth of bacteria and other pathogens, and promote a healthy environment; thus, they are crucial for propitious pregnancy and delivery. Neves et al. (17) demonstrated that lactic acid bacteria are characterized by a great demand for nutrients including amino acids and adenosine triphosphate. The production of adenosine triphosphate highly relies on glycolysis. A higher percentage 
of conditional pathogens leads to competition for glycolysis source metabolites with Lactobacilli; consequently, lactic acid production decreases and the vaginal $\mathrm{pH}$ increases to favor the growth of pathogenic species. As a result, the balance of the vaginal environment breaks down and the ascending genital infection heightens the risk of PROM. Interestingly, there is Lactobacillus supplement in forms of vaginal administration on the market, which in theory may help recover vaginal environment and benefit the pregnant women with bacterial vaginitis from development of PROM. However, we do not recommend this supplement before we get enough data to support its efficacy.

\section{Conclusions}

The integration of metagenomics and metabolomics is a powerful approach to researching the microorganism and metabolite functions in PROM. Metagenomics can elucidate the vaginal microorganism composition, which is involved in the development PROM, while metabolomics can uncover the biological functions of these microorganisms and their impact on local immune interaction. PROM develops as a result of the interaction of multiple factors, including the protective Lactobacillus species and vaginal dysbiosis, as well as the local host immune system. In the current study, we identified several microorganisms that may play a role in the development of PROM, including Streptococcus, Chlamydia, Prevotella, Staphylococcus, Mycobacterium, and Enterobacter. In addition, the protective role exerted by Lactobacilli via glycolysis and lactic acid production was confirmed from a biological perspective. The differences in GalNAc and sucrose, as upstream metabolites of glycolysis, between the PROM and HC groups further support our hypothesis. This study has demonstrated that the integrated screening of microorganisms and their metabolites, as an early warning system, is an effective approach to reducing the incidence of PROM and its related adverse pregnancy outcomes. The levels of GalNAc and sucrose, and the relative abundance of Lactobacillus versus conditional pathogens could serve as early indicators of PROM.

\section{Acknowledgments}

Funding: We thank the following funding agencies for their support of our work: Development and Demonstration Program of WuXi (grant No. N20192004); the 5th Phase of "Project 333" of Jiangsu Province of China (grant No. BRA2019024); Key Research and Development
Program of Jiangsu Province (grant No. BE2015617); the Key Research Program of Wuxi Commission of Health (grant No. Z202004); Innovation and Entrepreneurship Training Program for College Students in Jiangsu Province (KYCX19_1182); Wuxi Science and Technology Bureau (N20202031); and Jiangsu Province Maternal and Child Health Research Project (F202043).

\section{Footnote}

Reporting Checklist: The authors have completed the MDAR reporting checklist. Available at https://dx.doi. org/10.21037/atm-21-5539

Data Sharing Statement: Available at https://dx.doi. org/10.21037/atm-21-5539

Conflicts of Interest: All authors have completed the ICMJE uniform disclosure form (available at https://dx.doi. org/10.21037/atm-21-5539). The authors have no conflicts of interest to declare.

Ethical Statement: The authors are accountable for all aspects of the work in ensuring that questions related to the accuracy or integrity of any part of the work are appropriately investigated and resolved. This study was approved by the Ethics Committee of the Affiliated Wuxi Maternity and Child Health Care Hospital of Nanjing Medical University (No. 2019-02-0402-01) and was conducted in adherence with the Declaration of Helsinki (as revised in 2013). The study was retrospectively registered in the Chinese Clinical Trial Registry (ChiCTR2000034721, registration date: 2020-7-16). Written informed consent was obtained from each participant.

Open Access Statement: This is an Open Access article distributed in accordance with the Creative Commons Attribution-NonCommercial-NoDerivs 4.0 International License (CC BY-NC-ND 4.0), which permits the noncommercial replication and distribution of the article with the strict proviso that no changes or edits are made and the original work is properly cited (including links to both the formal publication through the relevant DOI and the license). See: https://creativecommons.org/licenses/by-nc-nd/4.0/.

\section{References}

1. Peelen MJ, Luef BM, Lamont RF, et al. The influence 


\section{Page 12 of 13}

of the vaginal microbiota on preterm birth: A systematic review and recommendations for a minimum dataset for future research. Placenta 2019;79:30-9.

2. Xia H, Li X, Li X, et al. The clinical management and outcome of term premature rupture of membrane in East China: results from a retrospective multicenter study. Int J Clin Exp Med 2015;8:6212-7.

3. Zhong N. Achieving Lower Preterm Birth Rates in China via Reductions in Iatrogenic Preterm Births. Am J Public Health 2019;109:1489-90.

4. Brown RG, Marchesi JR, Lee YS, et al. Vaginal dysbiosis increases risk of preterm fetal membrane rupture, neonatal sepsis and is exacerbated by erythromycin. BMC Med 2018;16:9.

5. Davidesko S, Wainstock T, Sheiner E, et al. Long-Term Infectious Morbidity of Premature Infants: Is There a Critical Threshold? J Clin Med 2020;9:3008.

6. De Seta F, Parazzini F, De Leo R, et al. Lactobacillus plantarum P17630 for preventing Candida vaginitis recurrence: a retrospective comparative study. Eur J Obstet Gynecol Reprod Biol 2014;182:136-9.

7. Chandiramani M, Bennett PR, Brown R, et al. Vaginal Microbiome-Pregnant Host Interactions Determine a Significant Proportion of Preterm Labour. Fetal and Maternal Medicine Review 2014;25:73-8.

8. Fettweis JM, Serrano MG, Brooks JP, et al. The vaginal microbiome and preterm birth. Nat Med 2019;25:1012-21.

9. Kanbayashi S, Sato Y, Taga A, et al. Positive vaginal culture at rescue cerclage predicts subsequent preterm delivery. J

Matern Fetal Neonatal Med 2018;31:1161-5.

10. Theodoridis G, Gika HG, Wilson ID. LC-MSbased methodology for global metabolite profiling in metabonomics/metabolomics. Trends in Analytical Chemistry 2008;27:251-60.

11. Paramel Jayaprakash T, Wagner EC, van Schalkwyk J, et al. High Diversity and Variability in the Vaginal Microbiome in Women following Preterm Premature Rupture of Membranes (PPROM): A Prospective Cohort Study. PLoS One 2016;11:e0166794.

12. Brown RG, Al-Memar M, Marchesi JR, et al. Establishment of vaginal microbiota composition in early pregnancy and its association with subsequent preterm prelabor rupture of the fetal membranes. Transl Res 2019;207:30-43.

13. Ravel J, Gajer P, Abdo Z, et al. Vaginal microbiome of reproductive-age women. Proc Natl Acad Sci U S A 2011;108 Suppl 1:4680-7.

14. Pruski P, Lewis HV, Lee YS, et al. Assessment of
Liu et al. Metagenomics and metabolomics analysis of PROM women

microbiota:host interactions at the vaginal mucosa interface. Methods 2018;149:74-84.

15. Hoh JK, Lappas M, Liu C, et al. Preterm birth rate and dilemma of preterm labor treatment in Asia. Placenta 2019;79:68-71.

16. Liu L, Xu HJ, Chen JL, et al. Detection of Vaginal Metabolite Changes in Premature Rupture of Membrane Patients in Third Trimester Pregnancy: a Prospective Cohort Study. Reprod Sci 2021;28:585-94.

17. Neves AR, Pool WA, Kok J, et al. Overview on sugar metabolism and its control in Lactococcus lactis - the input from in vivo NMR. FEMS Microbiol Rev 2005;29:531-54.

18. Vazquez F, Fernández-Blázquez A, García B. Vaginosis. Vaginal microbiota. Enferm Infecc Microbiol Clin (Engl Ed) 2019;37:592-601.

19. Pramanick R, Mayadeo N, Warke H, et al. Vaginal microbiota of asymptomatic bacterial vaginosis and vulvovaginal candidiasis: Are they different from normal microbiota? Microb Pathog 2019;134:103599.

20. Yamaguchi K, Ohashi K. Management of group b streptococcus-positive pregnant women at maternity homes in JAPAN: a questionnaire survey of compliance among midwives. Matern Health Neonatol Perinatol 2018;4:4.

21. Lin Y, Ye J, Luo M, et al. Group B Streptococcus DNA Copy Numbers Measured by Digital PCR Correlates with Perinatal Outcomes. Anal Chem 2019;91:9466-71.

22. Koppes DM, Vriends AACM, van Rijn M, et al. Clinical value of polymerase chain reaction in detecting group B streptococcus during labor. J Obstet Gynaecol Res 2017;43:996-1000.

23. Kanninen TT, Quist-Nelson J, Sisti G, et al. Chlamydia trachomatis screening in preterm labor: A systematic review and meta-analysis. Eur J Obstet Gynecol Reprod Biol 2019;240:242-7.

24. Marconi C, de Andrade Ramos BR, Peraçoli JC, et al. Amniotic fluid interleukin-1 beta and interleukin-6, but not interleukin-8 correlate with microbial invasion of the amniotic cavity in preterm labor. Am J Reprod Immunol 2011;65:549-56.

25. Zurek OW, Pallister KB, Voyich JM. Staphylococcus aureus Inhibits Neutrophil-derived IL-8 to Promote Cell Death. J Infect Dis 2015;212:934-8.

26. Rathore H, Rahman AJ, Salman M, et al. Frequency of Early-onset Neonatal Sepsis Following Prolonged Rupture of Membranes. Cureus 2020;12:e6864.

27. Akbarian $\operatorname{Rad} Z$, Esmaeilzadeh S, Haghshenas Mojaveri M, et al. Maternal Recto-Vaginal Organisms and Surface Skin 
Colonization in Infants. Iranian Journal of Neonatology 2018;9:14-9.

28. Ravel J, Brotman RM. Translating the vaginal microbiome: gaps and challenges. Genome Med 2016;8:35.

29. Nasioudis D, Beghini J, Bongiovanni AM, et al. $\alpha$-Amylase in Vaginal Fluid: Association With Conditions Favorable to Dominance of Lactobacillus. Reprod Sci 2015;22:1393-8.

Cite this article as: Liu L, Chen Y, Chen JL, Xu HJ, Zhan HY, Chen Z, Chen DZ, Xu ZF, Xu DX. Integrated metagenomics and metabolomics analysis of third-trimester pregnant women with premature membrane rupture: a pilot study. Ann Transl Med 2021;9(23):1724. doi: 10.21037/atm-21-5539
30. Spear GT, French AL, Gilbert D, et al. Human $\alpha$-amylase present in lower-genital-tract mucosal fluid processes glycogen to support vaginal colonization by Lactobacillus. J Infect Dis 2014;210:1019-28.

31. Srinivasan S, Morgan MT, Fiedler TL, et al. Metabolic signatures of bacterial vaginosis. mBio 2015;6:00204-15.

(English Language Editor: J. Reynolds) 
Supplementary

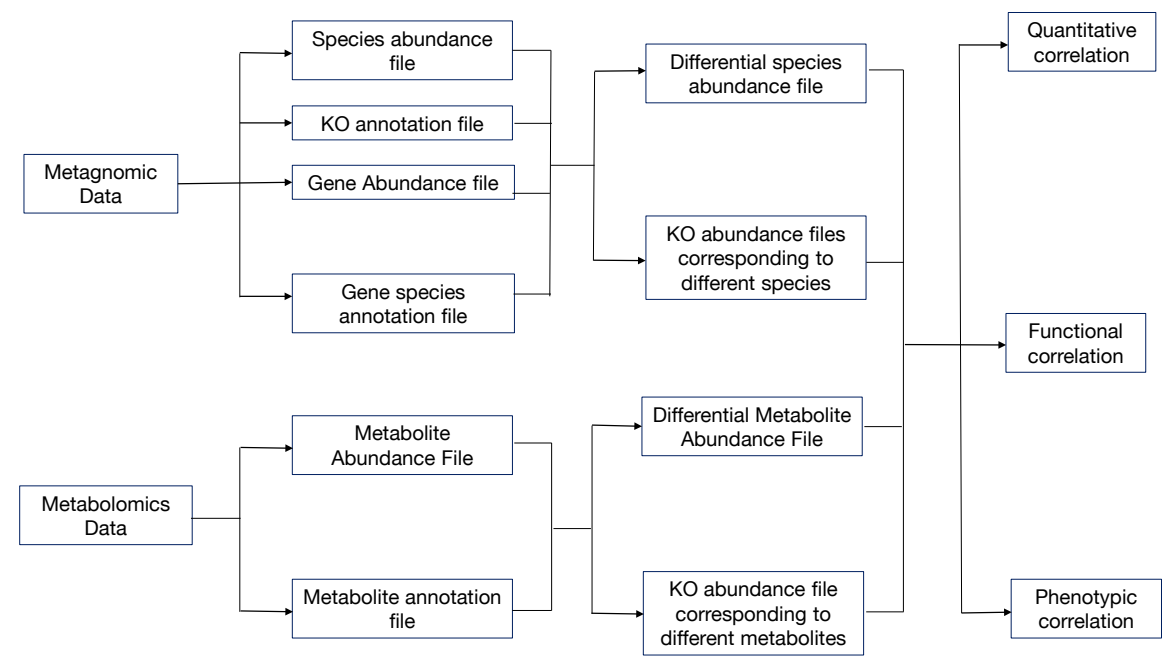

Figure S1 The flow chart of the metagenomics and metabolomics correlation analysis. 


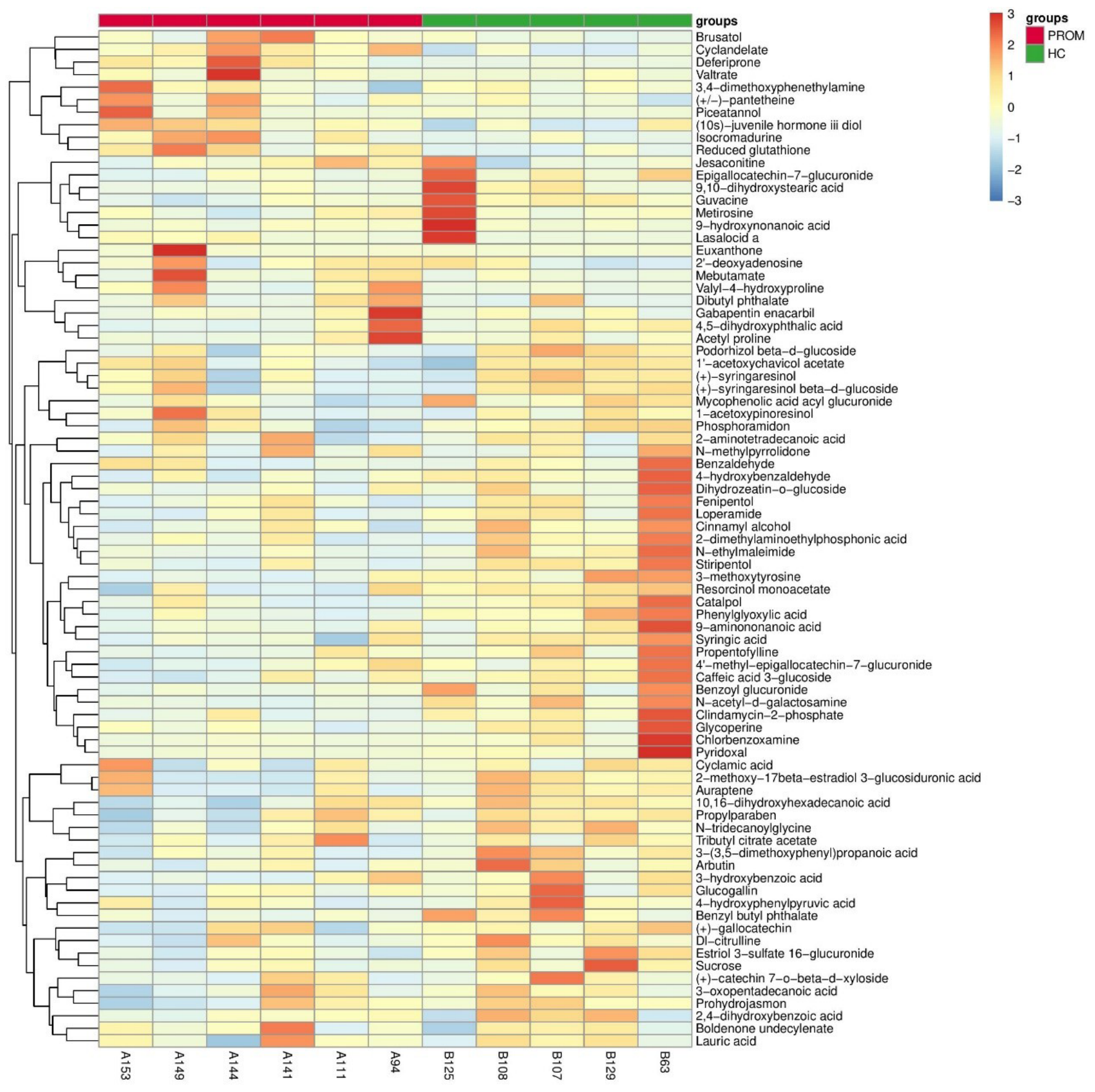

Figure S2 A heatmap of 82 differentially expressed metabolites between the PROM group and the HC group. PROM, premature rupture of membrane; HC, healthy control. 


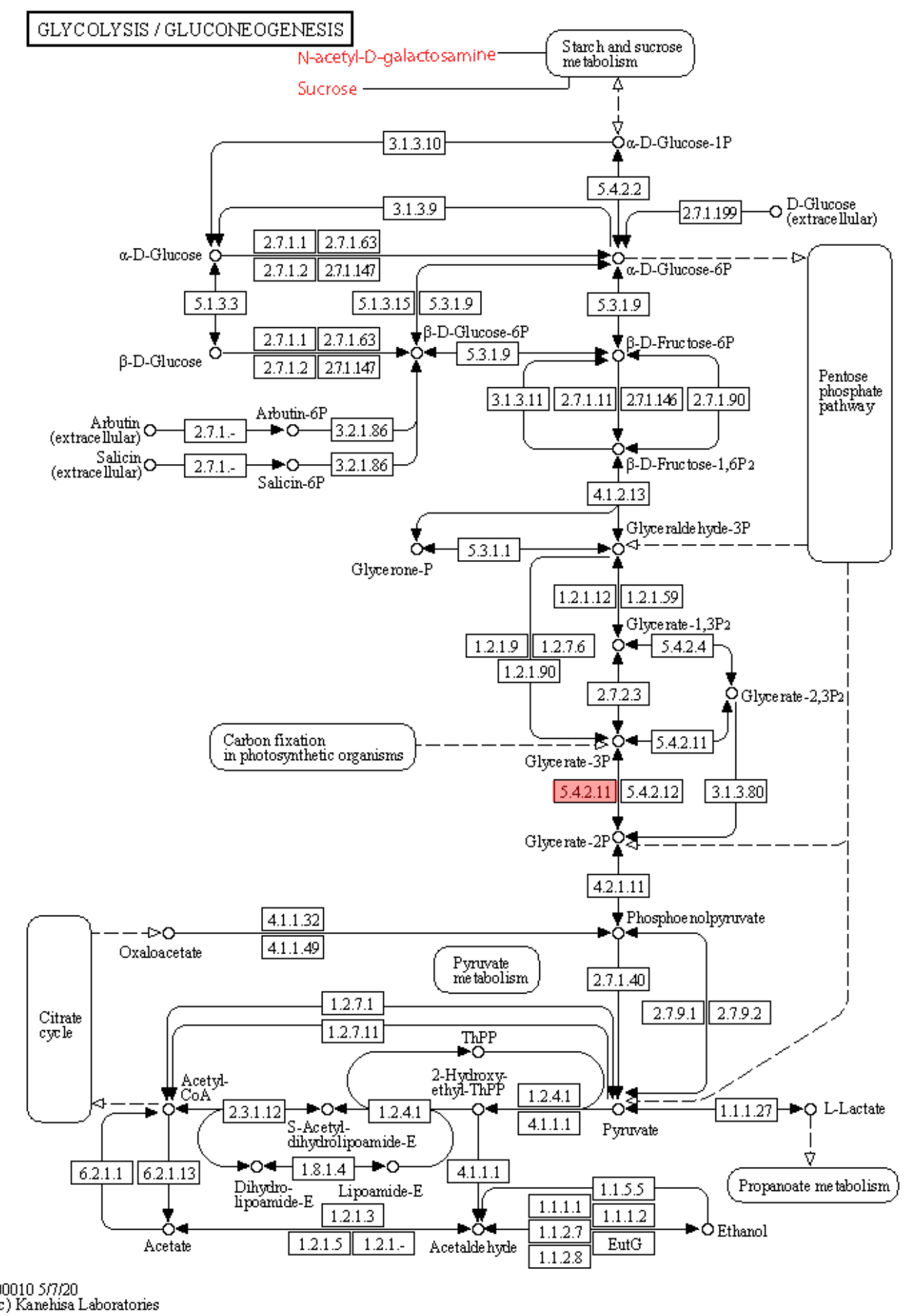

Figure S3 Diagram of the glycolysis pathway with key metabolites and enzymes. The glycolysis pathway was download from KEGG PATHWAY: Glycolysis/Gluconeogenesis-Reference pathway. 